\title{
Implementation of a siRNA Screen for Prostate Tumour Cell Lines Using Living Cell Arrays
}

\author{
Peggy Reich, Marcus Frohme, Juha Rantala, Matthias Nees
}

Zusammenfassung

Prostatakrebs ist neben Lungenkrebs eine der häufigsten Todesursachen bei Männern. In dieser Arbeit wurden 1500 potenziell tumorrelevante Gene in drei Zelllinien (VCaP, LAPC-4, RWPE-1) gescreent. Dafür wurde eine neue Technik, die Lebendzellarrays, genutzt, um Informationen über die Biologie Androgen-unabhängiger Prostatazellen zu gewinnen. Die Lebendzellarrays basieren auf dem Prinzip der reversen Transfektion [1], und in Folge werden die mRNAs der gewünschten Gene durch spezifische »silencer RNAs« (siRNAs) ausgeschaltet. Die Zellen wurden parallel sowohl in normalem Medium untersucht als auch mit androgen-reduziertem Medium unter Stress gesetzt. Das Wachstum der Prostatazellen wurde mittels Markern für Proliferation und Apoptose beobachtet. Die Daten der Screens wurden mit Hilfe statistischer Verfahren evaluiert. Die Lebendzellarrays konnten erfolgreich für einen umfangreichen SiRNA-screen angewendet werden.

\section{Abstract}

Prostate cancer is one of the most common causes of death in men. In this work a siRNA screen of around 1500 cancer-relevant genes was performed using 3 different cell lines (VCaP, LAPC-4, RWPE-1). A novel technique, the living cell array, was initiated in order to obtain information about the biology of Androgeninduced growth in prostate tumour cell lines. This technique is based on the principal of reverse transfection [1] and genes are knocked down by siRNAs. The cells on the living cell array were set under stress by reduction of the androgens in the media while the proliferation and apoptosis were quantified. The statistical analysis of the data implicates the success of the screen and shows that this method is suitable for large-scale experiments.

\section{Introduction}

Prostate cancer (PCa) is one of most common causes of death for men (figure 1). In many cases, this malignancy develops without apparent symptoms and is not discovered until metastases arise. In recent years, clinical tests measuring the release of PSA (prostate-specific antigen) into blood serum have significantly increased the number of cases detected at an early stage, resulting in much improved survival rates for these patients. Early diagnosis enables clinicians to successfully treat PCa using androgen ablation therapy [2]. However, particularly in advanced cases, the tumour recurs and develops androgen independent cells that are not responding to the ablation therapy, although almost invariably the androgen receptor (AR) continues to be expressed. This is illustrated by the fact that AR is very frequently amplified and overexpressed in hormone-refractory cancers (HRPCa), or acquires mutations that cause hyper-activation of its functions by very low amounts of ligands. Last not least, other mutations allow AR to bind unrelated, even non-steroidal ligands. It is this development of HR-PCa that poses a clinical problem for which, in fact, currently no therapy exists. It is important to understand this development of androgen independency to develop new therapeutic concepts and novel pharmaceuticals. Thus it is helpful to evaluate the network of relevant genes related to androgen-receptor signalling in order to model metabolic pathways and create hypotheses, which are then tested with specific experiments.

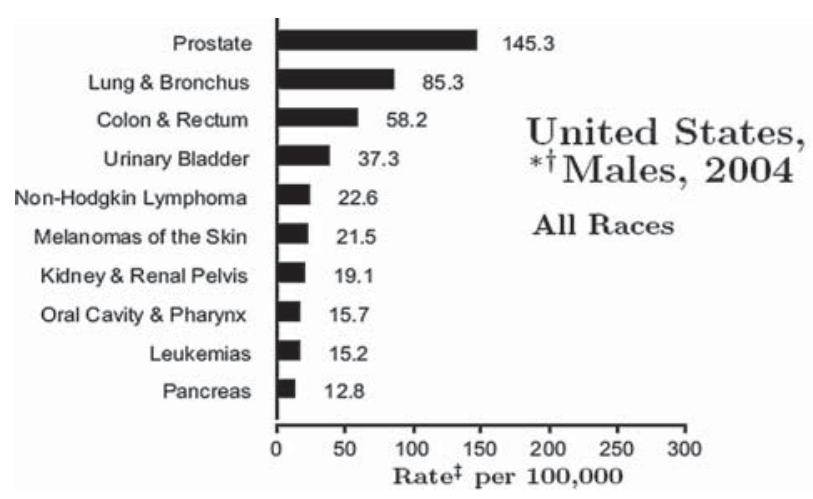

Fig. 1: Cancer cases (rated per 100000 persons) for males in the United States in the year 2004 [3] - data covers $98 \%$ of the population of the United States 
One of the key players in the male metabolism, Testosterone, is produced by the testes and the adrenal gland and converted in the prostate gland by $5 \alpha$-reductase to Dihydrotestosterone (DHT). The androgen receptor is located in the nucleus membrane and has a high affinity for this specific ligand DHT. The activated receptor dissociates from so called heat-shock proteins (HSP) and after dimerization and phosphorylation the androgen receptor (AR) binds specifically DNA sequences, i.e. the androgen responsive elements (ARE). With other co-activators the AR stimulates - as a transcription factor - proliferation and survival as well as it inhibits apoptosis of the prostate cells by regulating the transcription of androgen-responsive genes (fig. 2) [4]. Therefore prostate cells are strictly dependent on androgens to proliferate and survive. The reduction or removal of androgens in the cell culture is called androgen ablation. A prostate tumour may develop cells that are androgenindependent (hormone-refractory), meaning the cells found a way to proliferate by bypassing the normal signalling pathway. An androgen-independent prostate tumour will not respond to androgen ablation therapy and is the most common reason for cancer recurrence. The investigation of how these cells manage to stimulate proliferation without androgens is of outstanding importance.

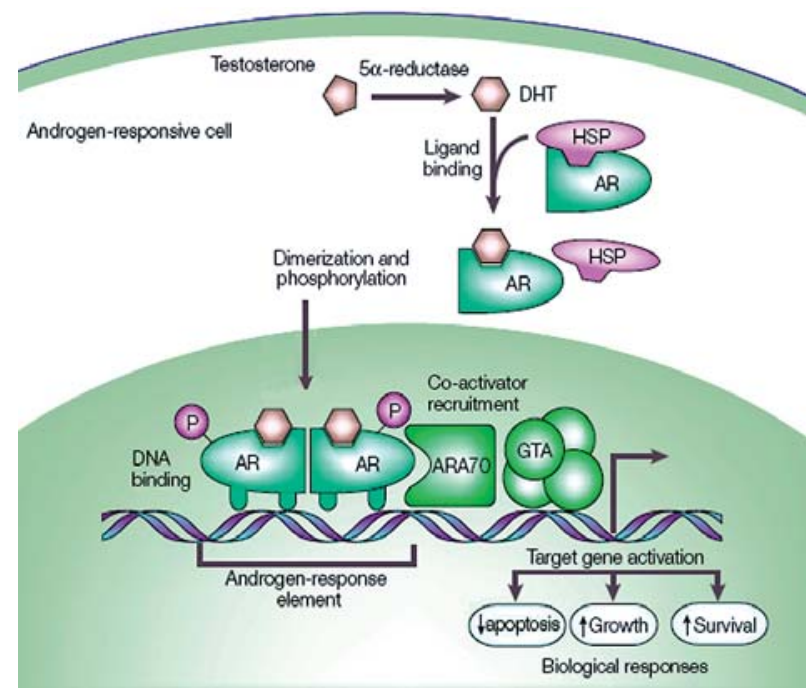

Fig. 2: The signalling pathway of the androgen receptor (AR). Testosterone is transported in the blood and taken up by the androgen responsive prostate cell. After transformation of testosterone to Dihydrotestosterone (DHT) by $5 \alpha$-reductase in the prostate cell, the DHT binds to the AR, which releases from the heat-shock-proteins (HSP). Then the AR is phosphorylated and dimerizes before it binds to androgen-response elements (ARA) on the DNA. With other coactivators like ARA70 and the general transcription apparatus (GTA) the AR regulates the transcription of genes to increase proliferation and survival of the prostate cells modified from [4].
This work is aimed to provide experimental data with information about the functions of genes in the prostate cell and the prostate tumour cell, with a special focus on genes in the context of AR functions and HR-PCa. Living cell arrays were used to screen a library of siRNAs. Additionally 2 cell lines (LAPC-4, RWPE-1) were established on living cell arrays, to enable comparison and validation of the data. The establishment includes investigation of the attachment behaviour of the prostate tumour cell lines. The cell arrays were kept under two different conditions: In media supplied with sufficient androgens and androgen ablated media.

\section{Materials and Methods}

For this study different cell lines were used: VCaP, LAPC-4 and RWPE-1, which were established from metastases of a human prostate carcinoma. The cell line VCaP was derived from a spinal cord metastasis of a PCa patient, and was initially maintained as mouse xenografts. The LAPC- 4 cell line was established from a lymph node metastasis of another PCa patient, which was also transplanted in xenografts. VCaP and LAPC-4 cells contain wild type AR and are androgen dependent (AD). Furthermore, VCaP contains a classical amplification of the AR locus on the $\mathrm{X}$ chromosome, causing AR to be highly expressed in this cell line. VCaP cells are strongly stimulated by very low amounts of androgens. In contrast, LAPC-4 does not contain an AR amplification and requires considerably higher concentrations of androgens to maintain growth and proliferation. By androgen ablation (= reducing/removing of androgens by the use of anti-androgens or charcoal-stripped serum) the cells die or exist without proliferation. RWPE1 is a normal prostate epithelial cell line which was immortalized using the oncogenes E6 and E7 of human papillomavirus type 18 (HPV18). In this study, RWPE-1 cells served as the normal control.

The living cell array (fig. 3) was developed in the research group »Medical Biotechnology« at VTT Turku (Technical Research Centre of Finland) [Rantala, manuscript in preparation]. Cell arrays are essentially based on the concept of reverse transfection, i. e. the transfection reagent is printed on a glass or plastic slide before the cells are added and left to attach. Recently, this experimental procedure has been described for small interfering (siRNA) arrays [1]. 


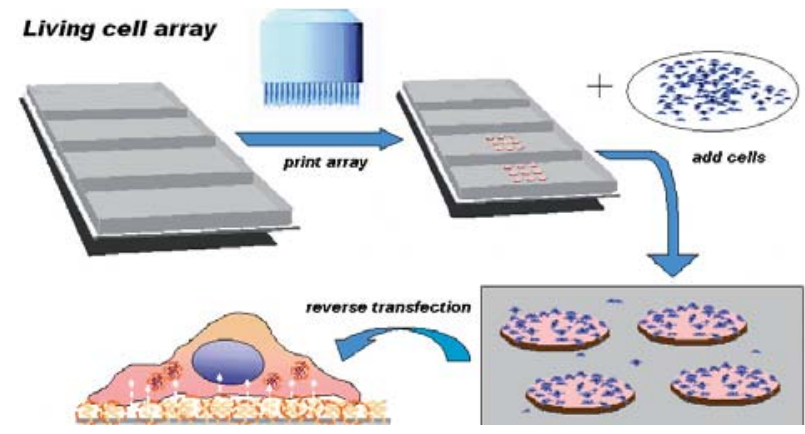

Fig. 3: The principle of a living cell array. First print transfection solution, including siRNA and attachment factors, on a plate. Next, cells are added and left to attach to the printed spots. The transfection of the cells on the spots is performed for $2-3$ days. After transfection, certain proteins of interest can be labelled by antibodies and assayed.

In vivo, these small RNAs interfere with the expression of a specific gene (this is also called RNAi = RNA interference). In vitro applied synthetic siRNAs for human cells are 19 to 25 nucleotides long, double stranded and have a small nucleotide overhang on both sides. It is mandatory for siRNAs to oblige to these size restrictions because long double stranded RNAs (> $30 \mathrm{nt}$ ) will effectively activate the interferon pathway which may lead to apoptosis and inflammation-like artifacts. To introduce siRNA into human cells, an effective transfection method is necessary, normally performed with polar lipids (e. g. lipofectamine [1]). Once introduced in the cell, in particular into the cytoplasm, siRNA knocks down specific genes of interest by silencing target mRNAs through degradation. The siRNAs bind to a complex named RISC, containing a number of cellular proteins that essentially accomplish binding of the siRNA to it's mRNA target molecule, causing a cleavage of the message followed by rapid degradation by other nucleases.

This refers to one limitation of RNAi screens in human cells: A gene may create many different mRNAs through splicing - thus many independent siRNAs per gene are required to efficiently knock down the gene [1]. Compared to the typically used liquid phase transfections in 96- or 384-well plates using robotics, the »reverse transfection « is an extremely powerful method for high-throughput screening of many genes in parallel to gain knowledge about their function. The high density of the spots allows an accelerated data acquisition and reduces the cost and resources for each experiment. In particular, the volume of siRNA required is decreased from several microlitres per well to only a few nanolitres per spot [1].

The layout of living cell arrays is variable and can be chosen individually.
The living cell array is printed in three steps (fig. 4). Every field is printed with a 16 pin print head. Three fields were printed in parallel on one slide. All three fields together are called array. One field contains 16 grids (= 784 spots). One grid contains 49 spots, 7 rows and 7 columns. One spot per grid is empty (no solution printed) as control and orientation support. The array includes 2352 spots. The spots have a diameter of 200 to $300 \mu \mathrm{m}$.

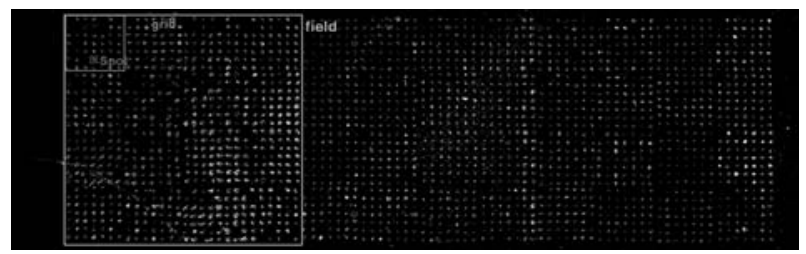

Fig. 4: Layout of a typical living cell array with VCaP cells. A cell array is divided in fields (in green), grids (in blue) and spots (in pink). In this approach, an array contains 3 fields each with 16 grids each with 49 spots. However, this is variable and depends on the printing strategy and the number of siRNAs printed.

The experimental design includes the selection of the siRNAs, controls, cell lines and conditions. The most time consuming part is to grow sufficient numbers of cells for seeding the arrays and to test their attachment capability. After the arrays were printed, the cells were attached, incubated, fixed and stained with antibodies. The staining was performed with an indirect immunoassay, using antibodies for ki67 and cParp. Ki67 is a protein present in the active cell cycle and widely used as a very reliable marker for cell proliferation. Cleaved Parp (cParp) is a proteolytically processed fragment of the poly-ADP-ribose-polymerase (PARP), which is cleaved during apoptosis. As fluorescent dyes, primarily the very stable fluorophores Alexa Fluor 488 and Alexa 555 were used. The cell »cores « were stained with SYTO, a red fluorescent dye that binds to DNA, thus highlighting the nuclei. The performed and stained arrays were subsequently scanned with a fluorescent laser scanner, and the intensities of the spots were measured with the Array-Pro ${ }^{\mathrm{TM}}$ Analyzer from Inc. Media Cybernetics. The spot intensities were automatically normalized using a program written in $\mathrm{R}$ [Kohonen $\mathrm{P}$, unpublished]. This algorithm first normalizes the variation per grid by dividing every intensity value of one channel by the median of the intensities in this channel per grid (fig. 5):

$$
y_{\text {kim }}=x_{\text {kim }} / \operatorname{median}\left(x_{i m}\right)
$$

Here $y_{k i}$ is the corrected intensity value and $x_{k i m}$ is the uncorrected raw value of the $\mathrm{k}^{\text {th }}$ spot in the $\mathrm{i}^{\text {th }}$ grid in the $\mathrm{m}^{\text {th }}$ channel, median is the median of the values in the $\mathrm{i}^{\text {th }}$ grid in the $\mathrm{m}^{\text {th }}$ channel. 

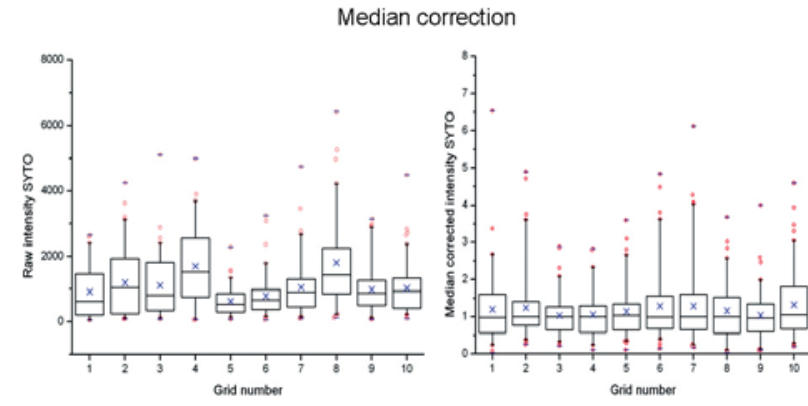

Fig. 5: Box chart of raw and median corrected intensities. The SYTO (= DNA dye) intensities for the first 10 grids of the VCaP cell array are displayed - left: raw intensity values; right: median corrected intensity values. The median of the grids form a line after correction. The box represents the median (horizontal black line) and the data values between the first quartile ( $25 \%$, bottom line of box) and third quartile (75\%, top line of box); the whisker (vertical lines from the box) are set from $5 \%$ to $95 \%$, the blue cross represents the mean of the data, the blue lines represents the maximal and minimal intensity values of the grid and the red dots represents the $10 \%$ outliers.

The intensities of the dyes vary over the array surface (fig. 6). In order to smoothen these variations in the dye intensities, the data are normalized using the loess approach (locally weighted polynomial regression, a.k.a. LOWESS) [6]. The three dye/antibody channels are ordered pairwise (cPARP/SYTO, Ki67/SYTO, Ki67/cPARP) based on their position on the plate and locally fitted by the loess function (fig. 7):

\section{Fit $=$ loess $($ rat $\sim$ int + grow + gcol $)$,}

with rat $=\log 2($ ChannelA/ChannelB $)$ and int $=\log 2$ (ChannelA*ChannelB) and grow the global row and gcol the global column of the array represent.
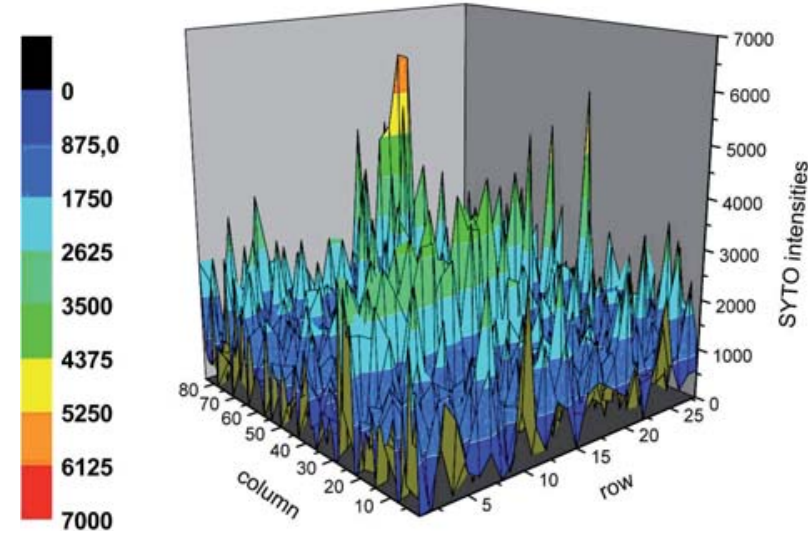

Fig. 6: Surface plot of a living cell array with VCaPs. The intensity of the stained nuclei using SYTO dye are plotted dependent on their location on the array. The array consists of 28 rows and 84 columns of spots. Local areas dominated by high SYTO intensities are clearly visible. These are smoothened using the 3-dimensional lowess correction.
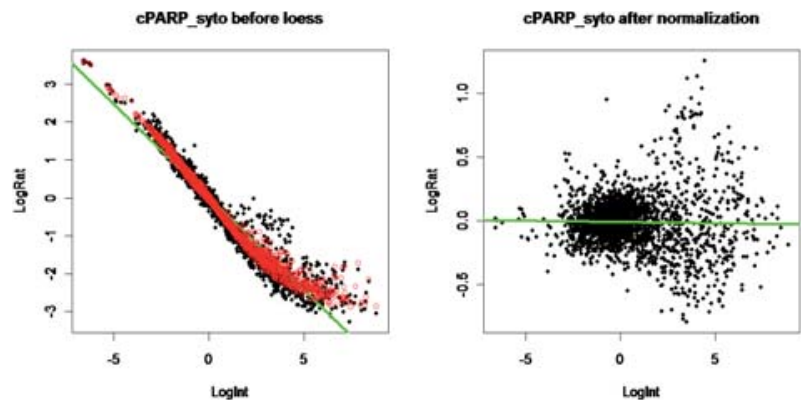

Fig. 7: Scatterplots of data values before and after the 3D-lowess-normalization. The channel pair CPARP/SYTO of the living cellarray with VCaP is displayed - left: Scatterplot of the median-corrected data before loess correction; right: Scatterplot of the with lowess corrected data - LogRat $=\log 2$ (int_CPARP/int_SYTO), LogInt = log2(int_CPARP*int_SYTO), black dots $=$ data values, grey dots $=3$ D-lowess fit, grey line $=$ linear lowess fit. The black dots represent the data values of the intensity ratio cParp/ Syto before lowess correction (in the left scatterplot) and after lowess correction (right scatterplot). The green lines represent the linear lowess fit without paying attention to the position of the intensities on the array. The red dots show the 3D lowess fit with attention to the position of the intensities on the array. The data values are corrected using the 3D lowess fit, the red dots.

\section{Results}

Several test plates with printed spots containing scrambled siRNA (i. e. siRNA without any information and no expected knock-down function) were used to define the conditions for the two cell lines LAPC-4 and RWPE-1. The conditions, time of enzyme treatment to detach the cells, the minimal cell amount needed as well as the time the cells require to attach were critically optimized and standardized conditions used to perform the actual screens.

The implementation and performance of the living cell arrays with the three cell lines VCaP, LAPC-4 and RWPE-1 was successful. Several other cell lines were also tested, incl. the widely used LNCaP cell line, but resulted in unsatisfactory data. The VCaP cells were left to attach for 45 minutes, while the LAPC- 4 cells were incubated for 25 minutes and the RWPE-1 cells were attached for one hour. All arrays were incubated for 36 hours in the appropriate medium, whereby the media was changed after 12 hours. Every array was fixed and stained, which required one full day per array. Eventually, the arrays were scanned and the obtained pictures were measured with the software Array-Pro ${ }^{\mathrm{TM}}$ Analyzer. This process is particularly time-consuming, since every spot has to be estimated individually and the automatic software tools are not sufficiently precise for this purpose. The normalization process itself is then automatically performed by the program. 
The spots were also observed using a fluorescence microscope (example illustrated in fig. 8). The detailed microscopic observation of hundreds of spots in principle confirmed that the antibody intensity correlates with the obtained values. Also, the SYTO-stained cellular nuclei are clearly visible, while the green fluorescence is situated surrounding the nucleus, which is expected for the Ki67 protein that is located in the cytoplasm.

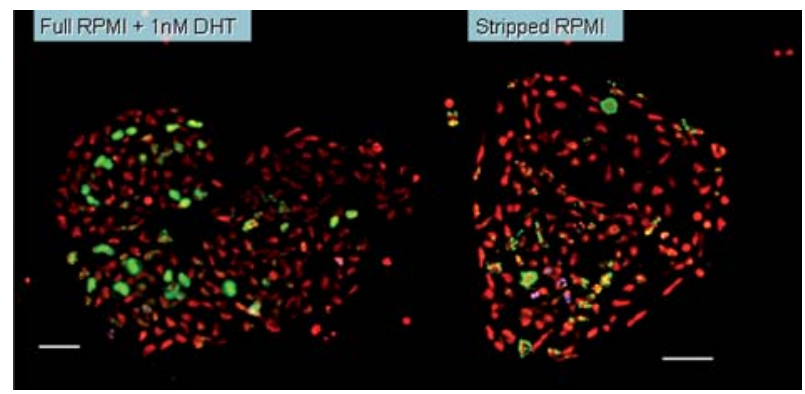

Fig. 8: Microscopic pictures of the VCaP array spot in full RPMI and stripped RPMI (without androgens) - white bar $=50 \mu \mathrm{m}$, red $=$ nuclear staining (SYTO), green = cell proliferation (ki67), blue $=$ apoptosis (CPARP). The apoptosis is more readily visibly in stripped, androgendepleted RPMI compared to full RPMI. In contrast, the proliferation/cell number ratio is roughly identical in both media

For every array one technical replicate was performed with the same cells, at the same time to estimate the random error of the arrays. The random error for VCaPs based on the normalized ki67/cParp intensity ratios (logarithmic) was calculated to be around $0 \pm 0.1$. Two thirds of the values per array lie inside this range. Only the values that are significantly higher or lower are truly interesting. The random error of the procedure is in a reasonable range. The reproducibility is good enough. To allow further evaluation of the data, some control siRNAs were used. Scrambled siRNAs contain no information and no gene-specific knock-down should occur. The calculated mean intensities are, as expected, within the normal range of variation $( \pm 0.1)$, indicating that transfection alone causes serious artificial effects. The performance of the antibodies was evaluated by a gene-specific knock-down of the mRNAs coding for the proteins those antibodies are expected to detect. The knock-down of the protein PARP (Poly-ADP-Ribose-Polymerase) decreased the intensity of the cParp (cleaved Parp) channel by more than $80 \%$. However, the knock-down of Ki67 decreased the intensity of the ki67 channel only by $5 \%$. This may be due to very high expression levels of $\mathrm{Ki} 67$ that are inefficiently targeted by the siRNAs, or, alternatively, indicating that the siRNAs against Ki67 are not working. For this reason, fur- ther controls such as MYC and KIF, whose knock-down was expected to cause cell death, were also used to control for transfection efficiency. Their knock-down decreased the intensity of the ki67/cParp ratio by around $50 \%$. This indicates that both the transfection of the cells and also the specificity of the antibodies used were satisfactory.

\section{Conclusion and future aspects}

The performance of the three living cell arrays with the cell lines VCaP, LAPC-4 and RWPE-1 was successful. The obtained data were also successfully evaluated and analyzed for biological relevance (data not shown here). A plate-to-plate normalization will allow the comparison of the intensities of two cell lines or two conditions by using statistical methods such as the student's t-test. These comparisons will produce additional functional information about the relevance of genes under the two different growth conditions examined. In particular, if the knock-down of a certain gene has no apparent growth effects in normal androgen-containing media but leads to increased cell death in androgen-reduced media, this may indicate a functional role of a gene in the androgen pathway. Most of the normalized data tend towards a somewhat higher variation, in particular at high intensities in both channels. Therefore an additional correction of the variance using loess will certainly improve the results.

\section{Annotation and Acknowledgement}

This work is based on the master's thesis of Peggy Reich (2008) [7] | Course of Studies Biosystemtechnik/Bioinformatics (BBM06). The major part of the experiments was done at the Technical Research Centre of Finland in Turku. 


\section{References}

[1] Erfle, H./Neumann, B./Liebel, U./Rogers, P./Held, M./Walter, T./ Ellenberg, J./Pepperkok, R. (2007): Reverse transfection on cell arrays for high content screening microscopy. In: Nature Protocols, Vol. 2, No. 2:392-399.

[2] Roach, M. (1997): III: Neoadjuvant therapy prior to radiotherapy for clinically localized prostate cancer. In: Eur Urol, 32 (suppl 3): 48-54.

[3] US Cancer Statistics Working Group: 2004 Incidence and Mortality. US Department of Health and Human Services, Centers for Disease Control and Prevention, National Cancer Institute; 2007.

[4] Feldman, B. J./Feldman, D. (2001): The development of androgen-independent prostate cancer. In: Nature Review, Vol. 1:34-45.

[5] Bokhoven, A./Varella-Garcia, M./Korch, C./Johannes, W. U./ Smith, E. E./Miller, H. L./Nordeen, S. K./Miller, G. J./Lucia, M. S. (2003): Molecular Characterization of Human Prostate Carcinoma Cell Lines. In: The Prostate 57:205-225.

[6] König, R./Chiang, C./Tu, B. P./Yan, S. F./DeJesus, P. D./Romero, A./ Bergauer, T./Orth, A./Krueger, U./Zhou, Y./Chanda, S. K. (2007): A probability-based approach for the analysis of large-scale RNAi screens. In: Nature Methods, Vol. 4, No. 10:847-849.

[7] Reich, P. (2008): Optimization of living cell array for functional characterization of prostate tumor cell lines. Master's thesis, University of Applied Sciences Wildau, Germany.

\section{Authors}

Peggy Reich, Master of Science

Technical University of Applied Sciences Wildau

Peggy_R@web.de

Prof. Dr. sc. hum. Marcus Frohme

Technical University of Applied Sciences Wildau

Faculty of Engineering/Industrial Engineering and Management

Molecular Biology and Functional Genomics

Tel. +493375 508-249

marcus.frohme@tfh-wildau.de

Juha Rantala, PhD student

Technical Research Centre of Finland, VTT Turku (Finland)

Medical Biotechnology, Biochip Technology

Juha.Rantala@vtt.fi

Matthias Nees, Senior Research Scientist

Technical Research Centre of Finland, VTT Turku (Finland)

Medical Biotechnology, Canceromics

Matthias.Nees@vtt.fi 